\title{
Long-Term Feeding of High Vitamin E Diet Improves the Decreased Mitogen Response of Rat Splenic Lymphocytes with Aging
}

\author{
Sakiyo SAKAI and Satoru MoriguchI* \\ Department of Nutrition, School of Medicine, The University of Tokushima, \\ Tokushima 770, Japan
}

(Received September 6, 1996)

\begin{abstract}
Summary This study was performed to investigate whether the longterm feeding of a high vitamin $\mathrm{E}$ (VE) diet has a beneficial effect on the decreased cellular immune functions caused by aging. Male Fisher rats, 12 weeks old, were fed a regular ( $50 \mathrm{mg} \mathrm{VE} / \mathrm{kg}$ diet) or high VE diet (585 $\mathrm{mg} \mathrm{VE} / \mathrm{kg}$ diet) for 12 months. Then, the rats were sacrificed under anesthesia and their cellular immune functions were measured. The proliferation of splenic lymphocytes with PHA or Con A was significantly lower in old rats fed the regular diet as compared to that of young rats (two months old). In contrast, the proliferation of splenic lymphocytes in old rats fed the high VE diet was similar to that of young rats. The in vitro effect of macrophages $(\mathrm{M} \phi)$ on the proliferation of splenic lymphocytes from young rats was investigated under Con A stimulation. Although splenic $\mathbf{M} \phi$ isolated from old rats fed the regular diet did not have any effect on the proliferation of splenic lymphocytes, $\mathbf{M} \phi$ from old rats fed the high VE diet significantly enhanced the proliferation of splenic lymphocytes. The responsiveness of splenic lymphocytes isolated from each group to the $\mathbf{M} \phi$ of young rats under Con A stimulation was not significantly different between the young rats and old rats fed the regular diet. In old rats fed the high VE diet, the responsiveness of splenic lymphocytes to young rat $\mathbf{M} \phi$ was significantly higher than that of the young rats or old rats fed the regular diet. Furthermore, the high VE diet induced a significant increase in interleukin 2 (IL2) production from splenocytes in both young rats and old rats following in vitro stimulation with Con A for $48 \mathrm{~h}$. These results suggest that VE has the ability to improve the decreased cellular immune functions caused by aging, and appears to be associated with the enhancement of both $\mathbf{M} \phi$ functions and lymphocyte responsiveness.
\end{abstract}

Key Words splenic lymphocytes, macrophages, mitogen response, interleukin 2, vitamin $\mathrm{E}$, aging, rats

\footnotetext{
* To whom correspondence should be addressed.
} 
Vitamin E (VE) acts as an antioxidant in cellular membranes and scavenges free radicals by blocking the peroxidation of polyunsaturated fatty acids $(1,2)$. In addition, it has been shown that VE also modulates immune responses. Tengerdy et al. reported that the dietary supplementation of VE leads to enhanced humoral immune responses and increased resistance to bacterial infection in mice and chicken $(3,4)$. Tanaka et al. found that the dietary supplementation of VE induces the enhancement of helper T-cell activity in mice (5). We have also reported that a high intake of VE increases the functions of splenic lymphocytes and alveolar macrophages (AM) in rats (6). These reports suggest the possibility that VE supplementation may improve the decrease of cellular immune functions caused by aging. However, there are not very many studies on the effect of VE supplementation on the decrease of cellular immunity caused by aging. Although Meydani et al. found that VE supplementation improved the decreased cellular immune functions in aged people (7), there is still little information on the effects of a high VE diet on the decrease of cellular immune functions caused by aging. In this study, we tried to investigate whether the long-term feeding of a high VE diet can improve the decrease of cellular immune functions caused by aging, and the mechanism of the role of macrophages $(\mathbf{M} \phi)$ in the mitogen response of splenic lymphocytes is discussed.

\section{MATERIALS AND METHODS}

Animals and diets. Specific pathogen-free, inbred male F344 rats, 12 weeks old, were randomly divided into two dietary groups: regular and high VE groups. Each group consisted of five rats. They were fed diets containing different levels of VE (50 and $585 \mathrm{mg} / \mathrm{kg}$ diet) (Table 1) for 12 months. Food and water were given with free access. Body weight and food intake were measured once a week during the experiment. The animals were sacrificed under anesthesia with sodium pentobarbital ( $0.1 \mathrm{ml} / 100 \mathrm{~g} \mathrm{BW}$, Abbot Laboratories, North Chicago, IL, USA) at 15 months old. Young male F344 rats, eight weeks old and weighing about $170 \mathrm{~g}$, were used as the control. The spleens of these animals were aseptically removed and weighed.

Splenocyte suspension. The spleen from each animal was minced with scissors and passed through a stainless steel mesh in RPMI 1640 culture medium (Gibco Laboratories, Grand Island, NY, USA). The number of splenocytes was counted microscopically and used for the following assays.

Proliferation of whole splenocytes. The responses of splenic lymphocytes to concanavalin A (Con A; $5 \mu \mathrm{g} / \mathrm{ml}$ ) and phytohemagglutinin (PHA; $10 \mu \mathrm{g} / \mathrm{ml}$ ) were determined as described previously $(8)$. Briefly, single-cell suspensions $\left(1 \times 10^{6}\right.$ cells $/ \mathrm{ml}$ ) were prepared in RPMI 1640 culture medium supplemented with $25 \mathrm{mM}$ 4(2-hydroxyethyl)-1-ethanesulfonic acid (HEPES; Sigma Chemical, St. Louis, MO, USA) $50 \mu \mathrm{M}$ 2-mercaptoethanol (2-ME; Sigma Chemical) and $2 \mathrm{mM} \mathrm{L-}$ glutamine, penicillin $(100 \mathrm{units} / \mathrm{ml})$, streptomycin $(100 \mu \mathrm{g} / \mathrm{ml})$ and $5 \%$ heat- 
Table 1. Composition of the basal diet.

\begin{tabular}{lc}
\hline \multicolumn{1}{c}{ Ingredients } & Concentration (\%) \\
\hline Vitamin E-free casein* & 20 \\
Sucrose & 10 \\
Cornstarch & 57 \\
Stripped corn oil & 8 \\
Mineral mixture** & 4 \\
Vitamin mixture $^{\#}$ & 1 \\
\hline
\end{tabular}

* Oriental Yeast, Tokyo, Japan.

\# Eisai Pharmaceutical Factory, Tokyo, Japan.

** The mineral mixture had the following composition, in $\mathrm{mg} / 100 \mathrm{~g}$ of mixture: $\mathrm{K}$, 420; P, 990; Na, 250; Mg, 74.9; Fe, 27.0; Zn, 5.1; Mn, 2.2; Cu, 0.57; I, 0.46.

\# The vitamin mixture had the following composition, in $\mathrm{mg} / 100 \mathrm{~g}$ of mixture: thiamine, 2.4; riboflavin, 8.0; pyridoxine, 1.6; cyanocobalamine, 0.001; ascorbic acid, 60.0; menadione, 10.4; biotin, 0.04; folic acid, 0.4; Ca-pantothenate, 10.0; $p$-aminobenzoic acid, 10.0; inositol, 12.0; niacin, 12.0; choline-chloride, 400.0; and $d l-\alpha$-tocopheryl nicotinate, 500.0. The following vitamins were also included, in $\mathrm{mg} / \mathrm{kg}$ diet: retinyl acetate, 0.03; and cholecalciferol, 0.005 . The high VE diet was prepared by adding $535 \mathrm{mg} d l-\alpha$-tocopheryl nicotinate per $\mathrm{kg}$ diet to this basal diet.

inactivated fetal bovine serum (FBS; GIBCO, Grand Island, NY, USA), respectively. Splenocytes with and without mitogens were plated in 96-well microtiter plates, incubated at $37^{\circ} \mathrm{C}$ in a humidified incubator with $5 \% \mathrm{CO}_{2}$ and $95 \%$ air for $72 \mathrm{~h}$, and then pulsed with $\left[{ }^{3} \mathrm{H}\right]$ thymidine (specific activity $25 \mathrm{Ci} / \mathrm{mmol}$, New England Nuclear, Boston, MA, USA). After $20 \mathrm{~h}$, the cells were harvested by an automated sample harvester (Flow Laboratory, Rockville, MD, USA). Radioactivity was determined by a liquid scintillation counter (LSC-703, Aloka Corp., Tokyo, Japan). The data are indicated as the stimulation index, which was calculated by assigning a value of 1 to the radioactivity of splenic lymphocytes cultured with medium and comparing this to the radioactivity of splenic lymphocytes from each group treated with mitogens.

Preparations of lymphocytes and macrophages (M $\phi$ ) from whole splenocytes. After whole splenocytes were dissociated by passing through a stainless steel mesh in RPMI 1640 culture medium, their suspension was cultured for $1 \mathrm{~h}$ at $37^{\circ} \mathrm{C}$ in a petri dish. Then, the nonadherent cells were collected by washing the dish three times with the culture medium. Adherent cells were scraped off from the dish by a rubber polishman. $\mathbf{M} \phi$ were identified by nonspecific esterase staining. As the result, over $84 \%$ of the adherent cells were $\mathrm{M} \phi$. The viability of isolated splenic $\mathbf{M} \phi$ was $>90 \%$ by trypan blue dye exclusion. The $\mathbf{M} \phi$ were adjusted to $1 \times 10^{5}$ cells/ml in RPMI 1640 culture medium. Non-adherent cells, mostly lymphocytes, were adjusted to $1 \times 10^{6} \mathrm{cells} / \mathrm{ml}$ in RPMI 1640 culture medium. In the case of measuring the effect of $\mathbf{M} \phi$ on the proliferation of splenic lymphocytes, $\mathrm{M} \phi$ isolated from each group were cultured with splenic lymphocytes from young rats for $72 \mathrm{~h}$. In the case of measuring the responsiveness of splenic lymphocytes to $\mathbf{M} \phi, \mathbf{M} \phi$ 
isolated from young rats were cultured with splenic lymphocytes isolated from each group for $72 \mathrm{~h}$. In both experiments, the ratio of lymphocytes and $\mathbf{M} \phi$ was $10: 1$, and lymphocyte proliferation was measured by the same way as described in "Proliferation of whole splenocytes."

Interleukin 2 (IL2) activity. The splenocytes $\left(1 \times 10^{6}\right.$ cells $\left./ \mathrm{ml}\right)$ isolated from each animal were cultured with Con A $(5 \mu \mathrm{g} / \mathrm{ml})$ for $48 \mathrm{~h}$. Then, the supernatants of these cultures were harvested and used for IL2 assay. IL2 activity in the supernatant was measured by the proliferation of IL2-dependent CTLL-2 cells (9). CTLL-2 cells $\left(5 \times 10^{3}\right)$ in a volume of $100 \mu 1$ consisting of $50 \mu 1$ RPMI 1640 culture medium and $50 \mu 1$ supernatant were added to each well. After the plate was incubated at $37^{\circ} \mathrm{C}$ for $48 \mathrm{~h}, 1 \mu \mathrm{Ci}$ of $\left[{ }^{3} \mathrm{H}\right.$ ] thymidine was added to each well and the plate was further incubated at $37^{\circ} \mathrm{C}$ for $24 \mathrm{~h}$. The incorporation of $\left[{ }^{3} \mathrm{H}\right]$ thymidine into the DNA of CTLL-2 cells was measured by a liquid scintillation counter (LSC703, Aloka Corp.). IL2 activity in the supernatant was expressed as units $/ \mathrm{ml}$ in comparison with the proliferation of CTLL-2 with murine recombinant IL2 (Genzyme, Boston, MA, USA).

Statistical analysis. The data in this experiment are written as $\mathbf{M} \pm \mathbf{S D}$, and experimental results were analyzed by Student's $t$-test.

\section{RESULTS}

Food intake, and body and spleen weights

The daily food intake of old rats was not significantly different between the regular and high VE diet groups throughout the experiment. The weight gain in old rats was also not significantly different in both the regular and high VE diet groups during the experiment. The final body weight of each group was about $470 \mathrm{~g}$ at the end of this experiment. The plasma VE level was significantly lower in old rats fed the regular diet as compared to those of both the young rats fed the regular diet and old rats fed the high VE diet. Spleen weight was slightly lower in the old rats as compared to that of the young rats (Table 2). In addition, the number of splenocytes was significantly lower in old rats fed the regular diet as compared to that of the young rats. In contrast, old rats fed the high VE diet did not show a significant decrease in splenocyte number as compared to the young rats, and maintained almost the same number as that of the young rats (Table 2).

Table 2. Body and spleen weights, and number of splenocytes and plasma vitamin E.

\begin{tabular}{lcccc}
\hline \multicolumn{1}{c}{ Groups } & $\begin{array}{c}\text { Body wt. } \\
(\mathrm{g})\end{array}$ & $\begin{array}{c}\text { Spleen wt. } \\
(\mathrm{g} / 100 \mathrm{~g} \mathrm{BW})\end{array}$ & $\begin{array}{c}\text { Splenocyte no. } \\
\left(\times 10^{-7} / 0.1 \mathrm{~g} \text { spleen }\right)\end{array}$ & $\begin{array}{c}\text { Plasma vitamin E } \\
(\mu \mathrm{g} / \mathrm{ml})\end{array}$ \\
\hline Young & $171 \pm 18.7$ & $0.28 \pm 0.03$ & $4.45 \pm 0.42$ & $7.82 \pm 1.62$ \\
Old-Regular & $469 \pm 52.4^{* * *}$ & $0.22 \pm 0.02^{*}$ & $2.92 \pm 0.32^{* *}$ & $3.75 \pm 1.81^{*}$ \\
Old-High VE & $473 \pm 46.7^{* * *}$ & $0.22 \pm 0.02^{*}$ & $3.65 \pm 0.39$ & $7.63 \pm 1.35$ \\
\hline
\end{tabular}

Significantly different from young rats: ${ }^{*} p<0.05,{ }^{* *} p<0.01,{ }^{* * *} p<0.001$. 


\section{Proliferation of whole splenocytes with PHA or Con A}

The proliferation of splenocytes with PHA or Con A was significantly lower in old rats fed the regular diet than that of the young rats $(p<0.001)$. In contrast, the proliferation of splenocytes in old rats fed the high VE diet was slightly lower in PHA stimulation and almost the same in Con A stimulation as compared to that of the young rats (Fig. 1).

In vitro effect of macrophages $(M \phi)$ on the proliferation of splenic lymphocytes with Con $A$

The in vitro effect of the $\mathbf{M} \phi$ isolated from the splenocytes of young and old rats on the proliferation of splenic lymphocytes from young rats was investigated under Con A stimulation. Although the proliferation of splenic lymphocytes was significantly increased by in vitro incubation with $\mathbf{M} \phi$ from young rats $(p<0.01)$, $\mathrm{M} \phi$ isolated from old rats fed the regular diet did not have any effect on the proliferation of splenic lymphocytes (Fig. 2). In contrast, $\mathbf{M} \phi$ from old rats fed the high VE diet significantly enhanced the proliferation of splenic lymphocytes $(p<$ 0.05), which was almost the same as that of the $M \phi$ from young rats.

\section{Responsiveness of isolated splenic lymphocytes to $M \phi$ from young rats}

The responsiveness of splenic lymphocytes isolated from young and old rats to the $\mathbf{M} \phi$ from young rats was investigated under Con A stimulation. There was no

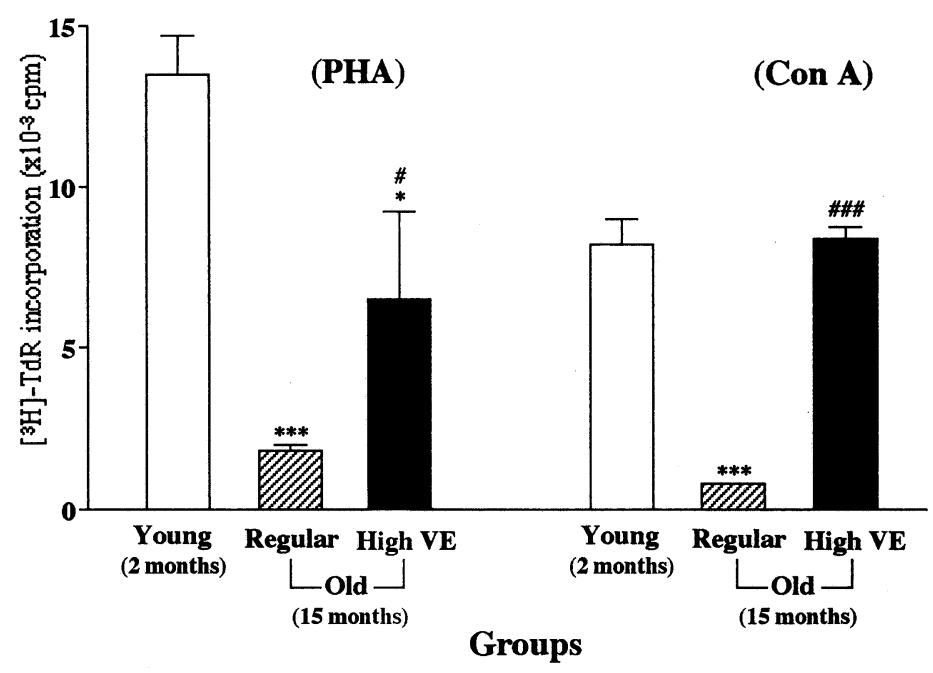

Fig. 1. The effect of a high vitamin $E$ (VE) diet on the proliferation of whole splenocytes with PHA or Con A. Splenocytes isolated from young rats and old rats fed regular or high VE diets for 12 months were incubated with PHA or Con $A$ for $72 \mathrm{~h}$. Then, the proliferation was measured by the uptake of $\left[{ }^{3} \mathrm{H}\right]-$ thymidine. Significantly different from young rats: ${ }^{*} p<0.05, * * * p<0.001$. Significantly different from old rats fed the regular diet: ${ }^{\sharp} p<0.05,{ }^{\#} p<0.001$. 


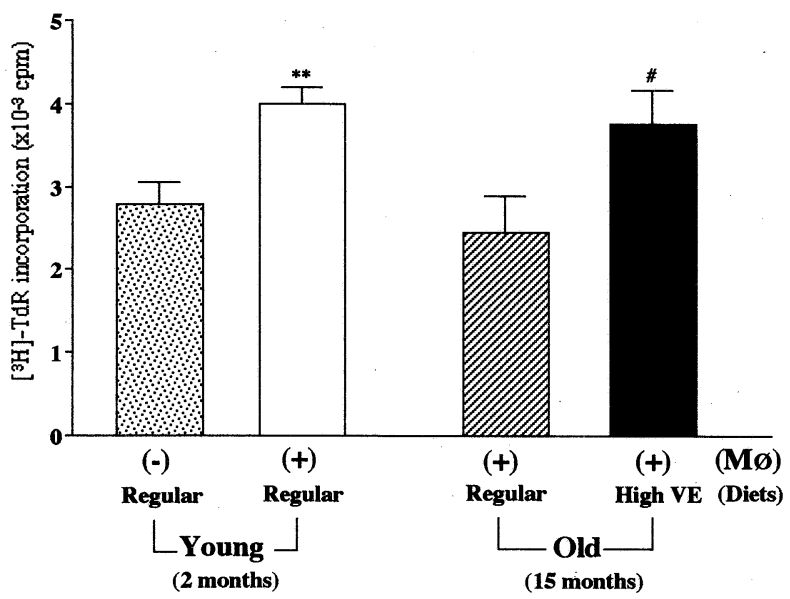

Fig. 2. The effects of aging and high vitamin $\mathrm{E}$ (VE) diet on the proliferation of splenic lymphocytes induced by macrophages $(\mathbf{M} \phi)$ under Con A stimulation. After M $\phi$ were isolated from the splenocytes of young and old rats fed the regular diet or old rats fed the high VE diet for 12 months, they were cultured with splenic lymphocytes isolated from young rats with Con A for $72 \mathrm{~h}$. The proliferation of splenic lymphocytes was measured by the uptake of $\left[{ }^{3} \mathrm{H}\right]-$ thymidine. $\mathbf{M} \phi(-)$, proliferation of splenic lymphocytes without $\mathbf{M} \phi ; \mathbf{M} \phi(+)$, proliferation of splenic lymphocytes with $\mathbf{M} \phi$. Significantly different from proliferation of young splenic lymphocytes without $\mathrm{M} \phi:{ }^{*} p<0.01$. Significantly different from proliferation of splenic lymphocytes in old rats fed the regular diet: ${ }^{\#} p<0.05$.

significant difference in the proliferation of splenic lymphocytes between the young and old rats fed the regular diet to $\mathrm{M} \phi$ (Fig. 3). In old rats fed the high VE diet, the responsiveness of splenic lymphocytes to $\mathbf{M} \phi$ was significantly higher than those of the young and old rats fed the regular diet $(p<0.05$ and $p<0.01$, respectively).

\section{Interleukin 2 (IL2) production from splenocytes}

IL2 production from splenocytes was significantly lower in old rats fed the regular diet as compared to that of the young rats $(p<0.05)$ (Fig. 4$)$. The high VE diet induced a significant increase of IL2 production from the splenocytes of old rats as compared to those of the young and old rats fed the regular diet $(p<0.05)$.

\section{DISCUSSION}

In this study, the long-term feeding of a high VE diet, 10 times higher than the regular diet, improved the decrease of cellular immunity caused by aging. As shown in Table 2, the decrease of the splenocyte number in old rats was prevented by a high VE diet. Since we have previously found that VE is an important factor 


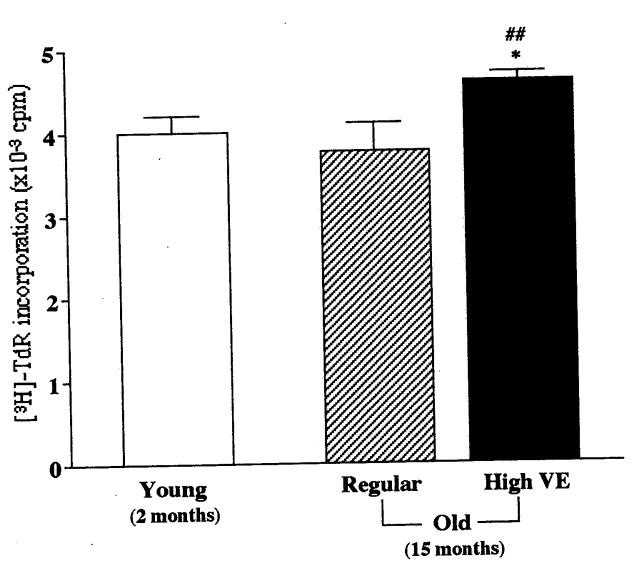

Fig. 3

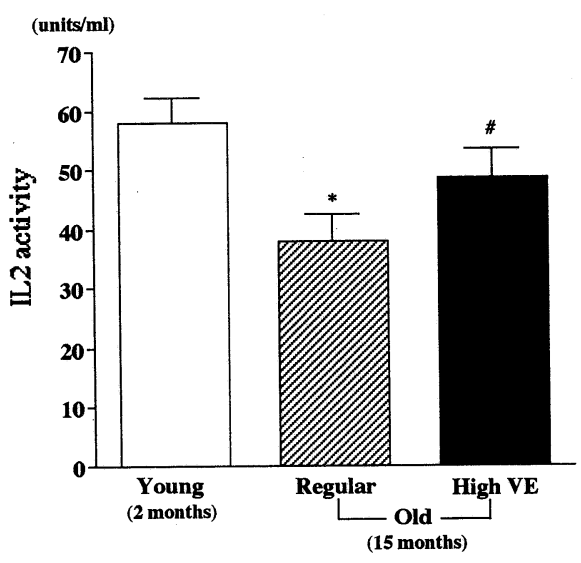

Fig. 4

Fig. 3. The effects of aging and high vitamin $\mathrm{E}$ (VE) diet on the responsiveness of splenic lymphocytes to macrophages $(\mathbf{M} \phi)$. After splenic lymphocytes were isolated from the splenocytes of young and old rats fed the regular diet or old rats fed the high VE diet for 12 months, they were cultured with $\mathbf{M} \phi$ isolated from young rats with Con A for $72 \mathrm{~h}$. The responsiveness of splenic lymphocytes isolated from each rat was measured by proliferation of its splenic lymphocytes to $\mathbf{M} \phi$ under Con A stimulation. Significantly different from young rats: $*_{p}<0.05$. Significantly different from old rats fed the regular diet: ${ }^{\#} p<0.01$.

Fig. 4. The effects of aging and high VE diet on the production of interleukin 2 (IL2) from splenocytes. After splenocytes were isolated from young and old rats fed the regular diet or old rats fed the high VE diet for 12 months, they were cultured with Con A for $48 \mathrm{~h}$. Then, IL2 activity in the supernatants was measured by proliferation of IL2-dependent CTLL-2 cells. Significantly different from young rats: $*_{p}<0.05$. Significantly different from old rats fed the regular diet: ${ }^{\#} p<0.05$.

for the differentiation and maturation of immature $T$ cells in the thymus of young rats $(10)$ and that it prevents the decrease of bone marrow cell proliferation after $\mathrm{X}$-ray irradiation (11), the long-term feeding of a high VE diet also stimulates the hematogenous function and may result in the induction of an increase of the splenocyte number in old rats. In addition, the decreased proliferation of whole splenocytes in old rats was largely or somewhat improved by the high VE diet as compared to that of the young rats. These results suggest that the increase of splenocyte number in old rats fed the high VE diet is due to the increase of mature $T$ cells, not immature $T$ cells. The long-term feeding of VE may induce higher differentiation and/or maturation of immature $T$ cells and a higher supply of immature $\mathrm{T}$ cells to blood from bone marrow even in old rats, which may result in the induction of an increase of the splenocyte number.

In old rats fed the regular diet, the proliferation of whole splenocytes was 
dramatically depressed. In general, since the proliferation of splenic lymphocytes with Con $\mathrm{A}$ is known to be due to joint action with both lymphocytes and macrophages $(\mathrm{M} \phi)(12)$, the decreased proliferation of splenic lymphocytes in old rats may be due to the impairment of lymphocyte responsiveness and/or $\mathbf{M} \phi$ functions to induce higher lymphocyte proliferation. Although $\mathbf{M} \phi$ isolated from the splenocytes of old rats did not have any effect on the proliferation of splenic lymphocytes from young rats as shown in Fig. 2, the responsiveness of the splenic lymphocytes from old rats was almost the same as that of the young rats (Fig. 3). This evidence suggests that the decreased proliferation of splenic lymphocytes in old rats fed the regular diet is due not to the decrease of lymphocyte responsiveness but the impairment of $\mathbf{M} \phi$ functions to enhance lymphocyte proliferation. We have also found that alveolar M $\phi$ (AM) from old rats maintain the ability to respond to the $\mathrm{M} \phi$ activating factor (MAF) produced by activated lymphocytes (13). However, the splenic lymphocytes of old rats showed a lower production of MAF following in vitro stimulation with Con $\mathrm{A}$ as compared to that of the young rats. Namely, even though the $M \phi$ of old rats maintain the ability to respond to MAF, the lymphocytes of old rats can not produce enough MAF to activate the M $\phi$, and consequently, old rats show decreased cellular immunity.

Meydani et al. found that the prostaglandin $\mathrm{E}_{2}\left(\mathrm{PGE}_{2}\right)$ level in blood was significantly increased in aged people, which is associated with decreased cellular immunity in the aged (7). $\mathrm{PGE}_{2}$ is known to decrease lymphocyte proliferation via increased intracellular cAMP (14). The decreased proliferation of splenocytes in old rats, as shown in Fig. 1, may be due to not only the decreased stimulation of $\mathrm{M} \phi$ to lymphocytes (Fig. 2) via cytokines such as interleukin 1 (IL1) but also the increased production of $\mathrm{PGE}_{2}$ from $\mathrm{M} \phi$.

VE supplementation from a young age improved the decrease of cellular immunity caused by aging as shown in Fig. 1. The effect of VE supplementation on the proliferation of splenic lymphocytes was strongly shown in Con A stimulation as compared to PHA stimulation. This result agrees with the report of Meydani et al. (15) and a previous report by us (6). There is no answer as to why VE specifically enhances lymphocyte proliferation with Con A. However, there seems to be three possibilities on the action of VE to lymphocyte proliferation with Con A: a) VE may stimulate specific $T$ cells, which can only respond to Con $A$; b) since it is known that Con A activates both lymphocytes and $\mathrm{M} \phi$, the action of VE on lymphocyte proliferation may be due to the increased M $\phi$ functions; and c) VE may directly act on $\mathrm{M} \phi$ and decrease the production of $\mathrm{PGE}_{2}$. Until now, there have been no studies investigating the in vitro or in vivo effects of $\mathrm{VE}$ on the proliferation of T-cell subsets (helper or suppressor T lymphocytes) with PHA or Con A. Most of the studies on VE and lymphocyte proliferation have shown that VE induces higher proliferation of lymphocytes with Con A stimulation than with PHA stimulation (6). Since Con A stimulates not only lymphocytes but also $\mathbf{M} \phi$, VE enhances $M \phi$ functions in in vitro treatment with Con $A$, which may further increase lymphocyte proliferation. As $\mathbf{M} \phi$ were not removed from the lymphocyte 
cultures in this study, the action of VE to the $\mathbf{M} \phi$ remains unclear. However, we have found that VE supplementation enhanced the phagocytic activity of AM (6) and that in vitro culturing with VE significantly enhances lymphocyte proliferation, which is not associated with the production of $\mathrm{PGE}_{2}$ from $\mathrm{M} \phi$ (12). Furthermore, $\mathrm{M} \phi$ isolated from old rats fed the high VE diet induced a higher proliferation of splenic lymphocytes from the young rat as shown in Fig. 2. These results suggest that VE has the ability to increase $\mathbf{M} \phi$ functions.

In contrast, there are many studies showing that VE supplementation decreases the $\mathrm{PGE}_{2}$ level in blood and the production of $\mathrm{PGE}_{2}$ from lymphocytes or $\mathrm{M} \phi(15,16)$. In this study, VE supplementation prevented the decrease of plasma VE level caused by aging, and retained the same plasma VE level as young rats (Table 2). This evidence may suggest the possibility that VE supplementation induces a higher level of plasma VE, which results in the decrease of $\mathrm{PGE}_{2}$ production. VE supplementation improved the decrease of splenic proliferation caused by aging, which may be associated with both increased $M \phi$ functions and the decreased production of $\mathrm{PGE}_{2}$.

It has been reported that $\mathrm{PGE}_{2}$ inhibits the production of IL2 from human peripheral blood lymphocytes (PBL) (17). In this study, IL2 production from splenocytes showed the same trend as the proliferation of whole splenocytes as shown in Fig. 1. Old rats showed a lower production of IL2 from splenocytes, which was significantly improved by VE supplementation and reached the level of young rats fed the regular diet. This suggests that VE modulates IL2 production. Since IL1 requires the expression of IL 2 receptor on the lymphocyte membrane and subsequently higher proliferation of lymphocytes is induced, IL1 production from M $\phi$ may be also increased by VE supplementation. In fact, we have previously found that IL1 production by rat AM is significantly enhanced by a high VE diet (6). This hypothesis may be also supported by the evidence that $\mathbf{M} \phi$ from old rats fed the high VE diet induced a higher proliferation of splenic lymphocytes as shown in Fig. 2.

Taken together, this study suggests that VE has the ability to improve decreased cellular immunity in the aged, which is associated with enhanced $\mathbf{M} \phi$ functions and increased responsiveness of lymphocytes. To elucidate the mechanism of VE on the improvement of decreased cellular immunity in the aged, further work is now in progress using aging models such as spontaneously hypertensive rats (SHR) or senescent accelerated mice (SAM).

\section{REFERENCES}

1) Tappel, A. L. (1972): Vitamin E and free radical peroxidation of lipids. Ann. N.Y. Acad. Sci., 203, 12-28.

2) Oski, F. A. (1977): Metabolism and physiologic roles of vitamin E. Hosp. Pract., 12, 79-85.

3) Tengerdy, R. P., Mathias, M. M., and Nockels, C. F. (1984): Effect of vitamin E on 
immunity and disease resistance, in Vitamins, Nutrition and Cancer, ed. by Prasad, $\mathrm{K}$. N., Karger, Basel, pp. 118-122.

4) Tengerdy, R. P., Heinzerling, R. H., Brown, G. L., and Mathias, M. M. (1973): Enhancement of humoral immune response by vitamin E. Int. Arch. Allergy Appl. Immunol., 44, 221-232.

5) Tanaka, J., Fujiwara, H., and Torisu, M. (1979): Vitamin E and immune response. Enhancement of helper $\mathrm{T}$ cell activity by dietary supplementation of vitamin $\mathrm{E}$ in mice. Immunology, 38, 727-734.

6) Moriguchi, S., Kobayashi, N., and Kishino, Y. (1990): High dietary intakes of vitamin $\mathrm{E}$ and cellular immune function in rats. J. Nutr., 120, 1096-1102.

7) Meydani, S. N., Barklund, M. P., Liu, S., Meydani, M., Miller, R. A., Cannon, J. G., Morrow, F. D., Rocklin, R., and Blumberg, J. B. (1990): Vitamin E supplementation enhances cell-mediated immunity in healthy elderly subjects. Am. J. Clin. Nutr., 52, 557-563.

8) Moriguchi, S., Kobayashi, N., and Kishino, Y. (1989): Effect of vitamin E deficiency on the functions of splenic lymphocytes and alveolar macrophages. J. Nutr. Sci. Vitaminol., 35, 419-430.

9) Moriguchi, S., Maekawa, K., Miwa, H., and Kishino, Y. (1993): Effect of vitamin E supplementation on cellular immune functions decreased with aging in spontaneously hypertensive rats. Nutr. Res., 13, 1039-1051.

10) Moriguchi, S., Miwa, H., Okamura, M., Maekawa, K., Kishino, Y., and Maeda, K. (1993): Vitamin $E$ is an important factor in T cell differentiation in thymus of F344 rats. J. Nutr. Sci. Vitaminol., 39, 451-463.

11) Moriguchi, S., Oonishi, K., Kishino, Y., and Umegaki, K. (1996): Vitamin E supplementation induced an early recovery of cellular immunity decreased following X-ray irradiation. Nutr. Res., 16, 645-656.

12) Oonishi, K., Moriguchi, S., and Kishino, Y. (1995): The role of macrophages in increased mitogen response of rat splenic lymphocytes following in vitro incubation with vitamin E. J. Nutr. Sci. Vitaminol., 41, 445-453.

13) Moriguchi, S., Yoshioka, A., Toba, M., and Kishino, Y. (1987): Studies on cellular immune functions in various nutritional state - On the fundamental research of nutritional manipulation in aging-. Bull. Phys. Fitness Res. Inst., 65, Suppl., 167-175.

14) Rincon, M., Tugores, A., Lopez-Rivas, A., Silva, A., Alonso, M., De Landazurim, M. O., and Lopez-Botet, M. (1988): Prostaglandin $E_{2}$ and the increase of intracellular cAMP inhibit the expression of interleukin 2 receptors in human $\mathrm{T}$ cells. Eur. J. Immunol., 18, 1791-1796.

15) Meydani, S. N., Meydani, M., Verdon, C. P., Shapiro, A. C., Blumberg, J. B., and Hayes, K. C. (1986): Vitamin E supplementation suppresses prostaglandin $E_{2}$ synthesis and enhances the immune response in aged mice. Mech. Ageing Dev., 34, 191-201.

16) Sakamoto, W., Fujie, K., Handa, H., Nishihara, J., and Mino, M. (1991): Vitamin E inhibits $\mathrm{PGE}_{2}$ and $\mathrm{O}_{2}^{-}$productions in rat peritoneal macrophages. Biochim. Biophys. Acta, 1074, 251-255.

17) Rappaport, R. S., and Dodge, G. R. (1982): Prostaglandin E inhibits the production of human interleukin 2. J. Exp. Med., 155, 943-948. 